\title{
COSI7: THE NEW CEA REFERENCE ELECTRO-NUCLEAR SIMULATION TOOL
}

\author{
G. KRIVTCHIK ${ }^{1}$ \\ ${ }^{1}$ CEA, DES, IRESNE, DER, SPRC, F-13108 Saint-Paul-lez-Durance, France. \\ guillaume.krivtchik@,cea.fr
}

\begin{abstract}
Nuclear fuel cycle scenario studies are used as a prospective analysis tool in order to provide stakeholders with decision aid. Nuclear fuel cycle simulation tools model the deployment of reactor fleets, the mass flows in the fuel cycle, and track the nuclear materials. The CEA has been developing for more than 30 years the nuclear fuel cycle tool COSI. The latest version, COSI7, was designed in order to model dynamic systems of ever-increasing complexity with improved user experience. The new developments include functionalities designed to improve the user experience as well as new physical models and post-processing capabilities. COSI7 supersedes COSI6 as the CEA reference nuclear fuel cycle simulation code starting January 2020.
\end{abstract}

KEYWORDS: COSI, fuel cycle simulation tool, scenario

\section{INTRODUCTION}

Nuclear fuel cycle scenario studies are used as a prospective analysis tool in order to provide stakeholders with decision aid. They aim to help decision-makers understand the impact of decisions in complex systems. A way to provide such help consists in modeling the whole nuclear fuel cycle system as a physical system. Nuclear fuel cycle scenario codes tackle such problems, and use modeling techniques to track inventories, mass flows, and interactions between the different facilities of the fuel cycle. For more than 30 years, the CEA Cadarache has been developing COSI, the CEA nuclear fuel cycle simulation (FCS) tool. The previous iteration of the software, COSI6 [1], written in Java and using the Discrete Event Simulation (DES), was released in 2006, and aimed to improve the simulation algorithms robustness and upgradability. Several intermediate versions of COSI6 were also generated, mostly in order to reduce the computing resources required by scenario simulation. For instance, COSI6 versions featuring parallel computing or surrogate models for depletion computations were released [2,3]. However, as COSI6 performance improved, scenarios simulations featured an increased level of operational detail and required always more fine-tuning of facilities and strategies, progressively shifting the time-consuming processes of making scenarios from simulation towards dataset generation and scenario results analysis. COSI7 is a complete re-write in C++ of COSI6, designed in order to tackle the new challenges induced by the ever-increasing complexity of the scenarios. COSI7 features many new functionalities aimed at refining the modeling capabilities while simplifying the dataset generation and scenario analysis tasks. While the general behavior of physical models and the DES formalism were preserved, the architecture of the code was fully re-designed to improve performance and maintainability. 
The new features of COSI7 can be broadly separated into two categories: improving the user experience and refining the physical models. Such features are presented hereafter.

\section{NEW FUNCTIONALITIES TO IMPROVE USER EXPERIENCE}

This section discusses the new COSI7 functionalities that were implemented to improve the user experience, during the intricately linked processes of dataset generation, scenario simulation and scenario results analysis. Most of these functionalities are related to the two COSI7 graphical user interfaces (GUI).

\subsection{Improved Expert GUI}

Decades-long experience of scenario-making at CEA Cadarache shows that a substantial proportion of the scenario-making process is spent generating datasets. Efforts were made to improve the COSI expert GUI in order to make the dataset more understandable, more flexible and to limit possibilities for input errors. Like the COSI6 GUI, the COSI7 expert GUI uses the bottom-up formalism: low-level objects, such as fuel types, are included in consecutive higher-level objects, such as batch types, reactor types, reactors and so on. In order to ease dataset browsing in COSI7, each higher-level object possesses hyperlinks directing to the associated lower-level objects. As in COSI6, all of the objects are accessible via a tree. Given the high number of objects included in a COSI7 dataset (usually several hundred objects), a dataset search engine was also included in the GUI. The user types a query in the always open search field and the GUI display the corresponding objects.

A log browser, containing all the dataset modifications sequence, was implemented, along with "Undo" and "Redo" buttons allowing the user to undo or redo dataset modifications. Dataset modification is now performed on-the-fly and does not require validation from the user anymore. "Previous" and "Next" buttons also are available and lead the user to previously or subsequently selected objects.

Finally, post-processing functions (user-defined visualization via graphs directly plotted in COSI7 and output file generation functions) used to be transient in COSI6. Such functions are now persistent and can be saved along with the simulations they are associated with.

\subsection{New "Decision-Maker" GUI}

Scenario studies frequently bear strategic and communication components. For instance, many studies aim at determining optimal dates for commissioning facilities, or evaluating long-term nuclear radiotoxicity and so on. As a consequence, managers of various levels usually take part in those studies. However, FCS tools are generally quite complex to use. A possible explanation for the FCS complexity is the sacrifice of user-friendliness for the sake of advanced modeling capabilities. Consequently, the interaction between managers and scenario studies usually require the intervention of expert FCS tool users to provide them with results. Such a process tends to be time-consuming for both parties, and is made especially difficult by the fact that "back-of-the-envelope" calculations tend to return wrong results because of non-trivial yet non-negligible physical phenomena (e.g. decay, fresh fuel reactivity equivalence), interactions (e.g. mixing material flows, multi-recycling), thresholds (e.g. empty stocks, batch-wise fuel management) and the dynamic nature of scenarios themselves.

Due to said phenomena, building an advanced fuel cycle scenario requires time and expertise. However, post-processing scenario results, or performing sensitivity studies on existing scenarios, which is often necessary to get a good grasp of scenarios, does not always require the insight of experts, and could be directly done by managers if an adapted tool was available. Consequently, providing managers with a tool specifically designed to answer such demands may prove itself useful by avoiding the interactions 
needing a low level of expertise (e.g. producing data spreadsheets) between experts and decision-makers, thus giving more time for interactions with higher expertise content.

The COSI7 decision-maker GUI, "COSI7-Quick", was designed to be such a tool: a user-friendly platform for understanding and analyzing pre-designed scenarios. COSI7-quick is based on top-down logic: the user primarily accesses top-level objects (e.g. reactors), and accesses the arborescence of lowerlevel layers (e.g. succession of loadings, batch types, fuel types etc.) from the top-level object. This formalism, although inefficient in the frame of scenario-making, is very intuitive in the frame of "scenario-grasping", i.e. the process of understanding a scenario modeled by other persons. COSI7-quick is not designed to generate scenarios from scratch, but to analyze and understand scenarios made with the expert GUI.

COSI7-Quick includes safeguards: the user cannot access "expert" parameters such as the computation scheme, the scenario structure (front-end and back-end connections), or the physical models. Such parameters are only accessible from the expert GUI. However, many "fuel cycle strategy" options are available, including adding or removing reactors, modifying their operational parameters (commissioning, load factor, etc.), changing their loading while respecting a pre-established fuel cycle archetype and so on.

Any COSI7 simulation dataset can be accessed via both GUIs. COSI7-Quick shares the same simulator with the expert GUI, and its datasets bear no additional physical simplifications: simulating a dataset using a GUI will provide the exact same result as with the other.

In addition to interactive graph plotting, the COSI7-Quick GUI also brings a new, visual approach to the scenario simulation post-processing: the "fuel cycle" window brings in a single diagram all of the reactors, fuel cycle facilities and materials storage, and acts as a post-processing platform. The user can easily access the mass flows between the facilities (total, only plutonium, only minor actinides etc.), integrated or averaged, over any period of interest. Fig. 1 shows the "fuel cycle" window for a fuel cycle of interest. In this case, the fuel cycle diagram illustrates the facilities of a PWR UOX - PWR MOX SFR fuel cycle, as well as the associated plutonium mass flows (yellow boxes) integrated over a given period. The average electricity production per reactor over the same period is also shown (blue boxes).

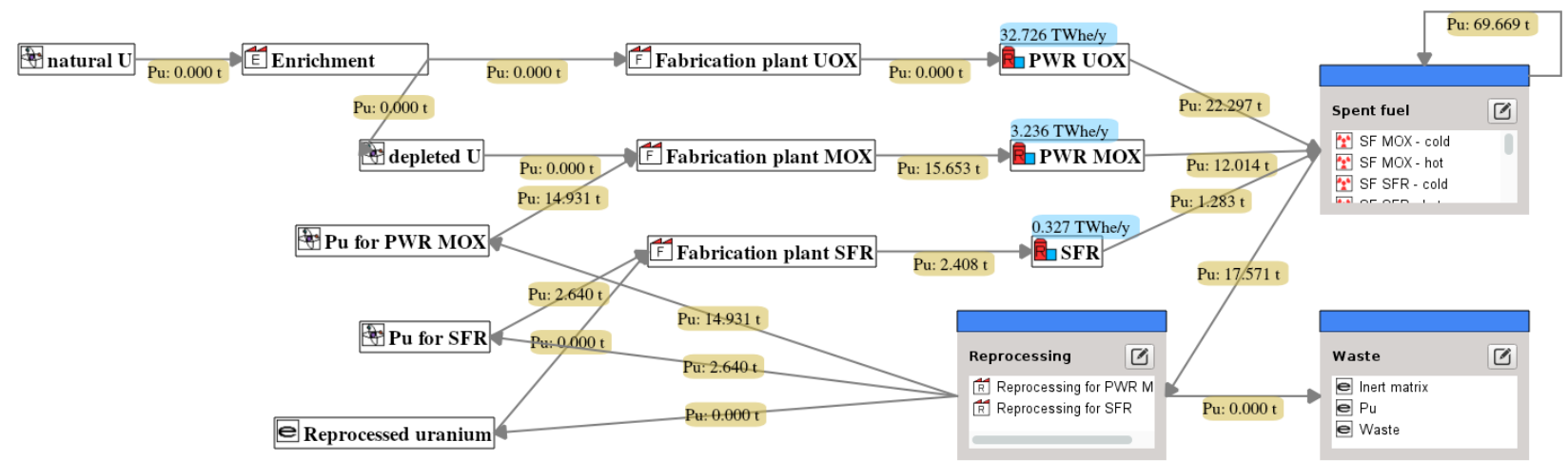

Figure 1. "Fuel cycle" window in the COSI7 decision-maker GUI

The facilities not in operation for the period of interest are greyed out. The user can dynamically group facilities together to reduce clutter, or separate them from groups in order to study them specifically.

\subsection{Streamed Post-Processing}

The scenario-making process can be highly iterative, for instance in the frame of multiple recycling strategies with numerous material flows. Such complex scenario computations can be time-consuming, 
especially when using precise physical models such as CESAR5.3 [4] for depletion. In order to shortcircuit the usual simulation / post-processing / analysis / dataset modification / simulation feedback loop, the streamed post-processing of ongoing simulations was made available in COSI7. If selected by the user, various curves are produced directly during the simulation process. These curves can be detached from the main window to be organized as a panel. Moreover these windows are persistent: if the user modifies the dataset and re-runs the simulation, the windows are automatically updated with the results of the new simulation. As a consequence, the post-processing functions can be defined once and for all, while the "analyze" step can be performed during simulation.

Post-processing uses a low-priority thread. As such it does not slow-down the scenario run.

\subsection{Server Computation and Shared Server Cache}

Simulating scenarios, especially using detailed physical models, is CPU-intensive. COSI7 is based on Discrete Event Simulation (DES) of batch-wise mass flows; said events being any operation (cooling, depletion, transportation etc.) performed on any batch of matter. The COSI7 client-server architecture was implemented in order to run COSI7 on low-resource computers while the results are streamed from higher end workstations. If the client is connected to a distant host via the ssh protocol, an option in the GUIs enables the user to run all CPU-intensive computations (depletion, evolution) of the simulation and the post-processing on the server. Both COSI7 client and server are compatible with parallel computing.

The COSI7 server includes an additional feature: a shared cache. The use of a cache had been previously introduced in COSI6: all of the depletion and evolution computations and their outputs are cached; if the same computation is called again then the outputs are recovered from the cache. Such a cache has been implemented in COSI7, both in the client and the server. Several cache settings are available, including:

- Disable cache (useful for low-RAM hosts connected to a server);

- Clear the cache;

- Limit the cache size (useful for low-RAM hosts and continuous operation servers).

The cache is especially convenient when a client-server approach is used: the server cache acts as a shared cache between the different server users. A user can benefit from the depletion and evolution runs stored by other users, including the same user using a previous client instance. For example, if a user runs a scenario simulation that has already run before, then the subsequent run is greatly facilitated by the fact that it requires no new depletion or irradiation computations as they are already stored in the server.

The interaction with the server was made such that server use is transparent for the end user and requires no specific knowledge of communication protocols. However, advanced users can monitor and manage the server via a dedicated channel.

\subsection{OS Compatibility and Setup Procedure}

COSI7 runs both on Windows and Linux. COSI7 setup (linking to external codes, etc.) is made directly from the GUIs and requires no script editing. Paths are tested before being saved in order to reduce human errors occurrence, and are tested again before new simulations.

\section{NEW PHYSICAL MODELS}

Scenarios modeled at CEA are becoming increasingly complex while requiring always more physical accuracy and industrial fidelity. This section details some of the new physical models that were introduced into COSI7 in order to provide more options to fine-tune scenarios and refine the physical description of facilities and processes. 


\subsection{Anticipation of Fresh Fuel Fabrication}

In COSI, fuel fabrication is dictated by fuel loading in reactors: if a given batch must be loaded at date $t$, then the associated fabrication plant receives the order to fabricate at date $t-d, d$ being the fabrication process duration. However, as reactors' loadings are asynchronous, even at steady state the fuel fabrication needs are not constant over time. Consequently, the fabrication plants generally have highly irregular production patterns. Such a result does not reflect the economic optimum: some years the fabrication plant will produce much below capacity. In COSI7, fuel fabrication anticipation was developed in order to streamline the fabrication profile. The user defines the fabrication capacity profile. If for a given period, the capacity is below the needs, then the fabrication of some batches is anticipated in the previous periods. Consequently, a certain amount of fresh fuel is not directly transferred from the fabrication plant to the reactor, but waits in a fresh fuel buffer stock. However, the waiting time is preevaluated, and the associated ageing of the fuel materials, taken into account for the fresh fuel fabrication equivalence model, is taken into account. For instance, if a PWR MOX fuel batch fabrication has to be anticipated, then its plutonium content will be higher than if not, in order to take into account the additional ${ }^{241} \mathrm{Pu} \rightarrow{ }^{241} \mathrm{Am}$ decay, while in the buffer, and the subsequent impact on fuel reactivity.

The anticipation algorithm itself is based on a backwards temporal scan of the fabrication needs which pushes further backward the needs if peak capacity is reached. The algorithm behavior is illustrated in Fig. 2. Two cases are presented, based on the same fuel fabrication need: the "infinite" case, in which fuel fabrication plant has no constraint; the "anticipation" case, in which the yearly capacity constraint is 250 t. The left side graph shows the batch-wise fuel fabrication: the use of anticipation at the fabrication plant produces smoother histories and avoids peaks: given the possibility to anticipate fuel fabrication by a up to one year, it is possible to reduce the capacity of the fabrication plant, defined by the peak capacity, by more than 35\%. The right side graph shows the associated fresh fuel stock inventory. The fresh fuel stock, which acts as a buffer between fabrication and loading, absorbs the variability of fresh fuel demand. It is null in the case of an infinite fabrication plant, but can be non-null in the case of anticipation.
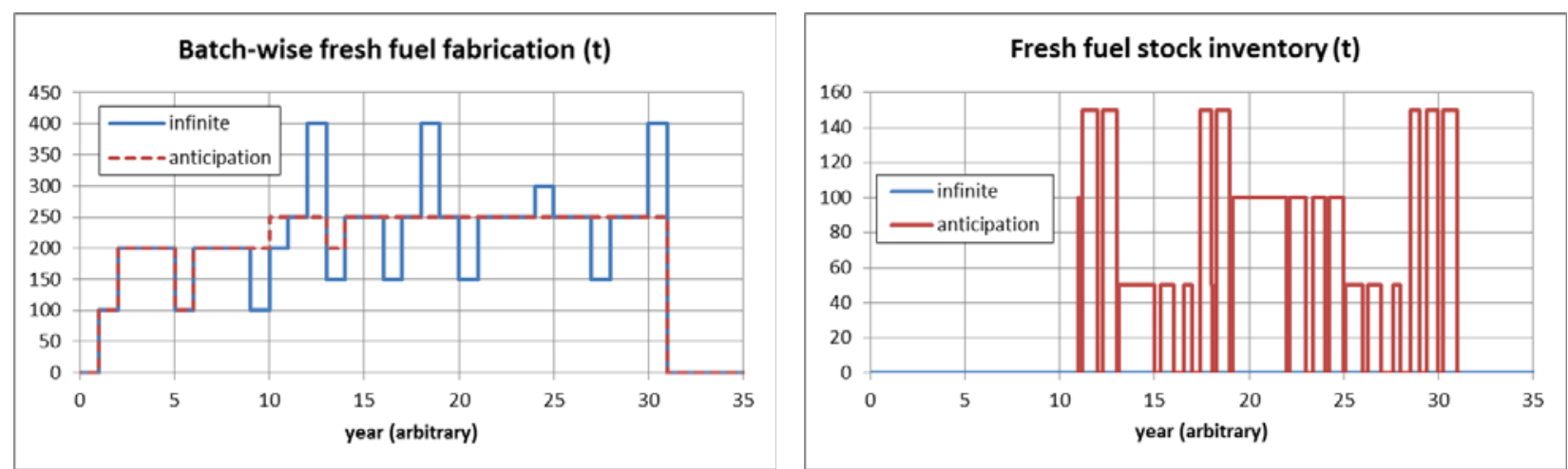

Figure 2. Impact of anticipation of fuel fabrication model

The fuel fabrication anticipation model can anticipate fuel fabrication for more than one year if necessary. It is also parameterized in terms of rate: the user can choose to divide years of operation into any number of sub-periods, each sub-period associated with a fixed capacity and the possibility to anticipate fuel fabrication.

It can be noted that in COSI7, the fabrication plants and the reprocessing plants can be individually modeled as passive plants responding to demands from the rest of the fuel cycle, or as active plants pushing materials into a buffer stock (i.e. fabrication plant using the "anticipation of fabrication" model, or reprocessing plant operating at a pre-defined capacity). 


\subsection{Spent Fuel Cooling Time Tracker}

Spent fuel (SF) cooling time is a relevant indicator to understand the dynamics of fuel cycles: it directly impacts the isotopic composition of materials through decay, and is an indicator of the amount of idle materials in the fuel cycle. When complex reprocessing strategies are applied (e.g. mixing first-in first-out and last-in first-out flows in non-equilibrium scenarios), inferring the cooling time from the mass flows is not easy. To overcome this difficulty, a SF cooling time tracker was implemented in COSI7. This module tracks the cooling time of every SF batch, and is used to generate both distributions and averages of cooling times, for each operation of the SF stock or each year of the scenario. The cooling time can be evaluated both as a balance (i.e. the cooling time of the SF moving in or out of stock) and as an inventory (i.e. the cooling time of the $\mathrm{SF}$ contained in a stock at a given time).

Fig. 3 illustrates the results of the SF cooling time tracker on a toy scenario with PWR UOX, PWR MOX and SFR reactors. In this scenario, spent fuel management is first-in / first-out (FIFO), and the minimal cooling time before reprocessing is 5 years. The reprocessing strategy is as follows:

- For PWR MOX fuel fabrication: Pu is separated from UOX SF only;

- For SFR fuel fabrication: Pu is separated from SFR SF if available. Otherwise it is separated from MOX SF.

The left side graph shows the SF inventories per batch type (UOX, MOX and SFR). The right side graph shows the average cooling time of SF being reprocessed. The SF ageing approximately follows the trends of the SF inventory. This result is intuitive for a scenario with a stationary fleet for which reprocessing plant uses FIFO SF management: the higher the SF inventory is, the older SF is at the moment of reprocessing. As SFR SF is reprocessed as soon as cooled enough (5 years in this case), the cooling time of SFR SF at reprocessing is always equal to 5 years. In constrast, MOX SF tends to pile-up: as a consequence, the cooling time tends to increase indefinitely.
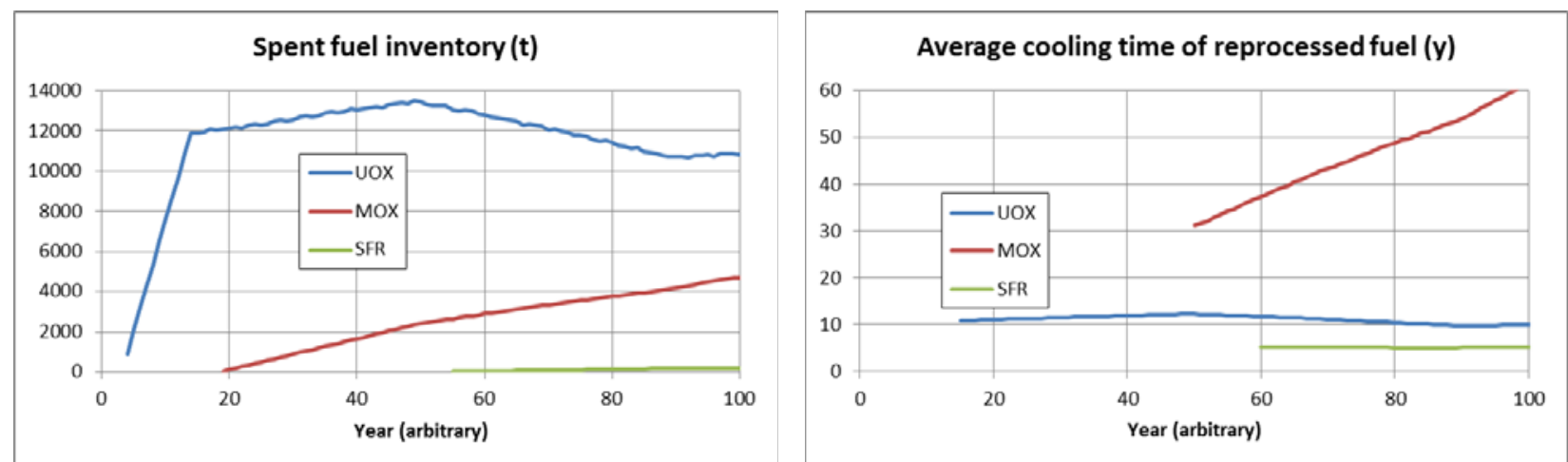

Figure 3. Spent fuel inventory and cooling time at reprocessing

\subsection{A Fresh Approach to Recipes}

Several fuel cycle simulation codes, including COSI, have a function sometimes referred to as "recipe". Recipes usually imply, during the process of mixed fuel fabrication, mixing two material flows with a given ratio. Such an approach is generally considered low-fidelity because it does not take into account the isotopic composition of the materials. In COSI6, fresh fuel fabrication preferably relies on so-called "equivalence models" which evaluate the U enrichment or Pu content (or other, depending on the fissile material) in function of the isotopic vector of the materials available, in order to satisfy an equivalence criterion (e.g. beginning of cycle or end of cycle neutronic reactivity). Due to variability in the isotopic composition of available materials (e.g. separated $\mathrm{Pu}$ originating from various spent fuel assemblies), fluctuations in the fresh fuel enrichment or Pu content are generally encountered. Some specific fuel 
designs are reliant on keeping the fresh fuel $\mathrm{U}$ enrichment or $\mathrm{Pu}$ content within a limited domain, possibly conflicting with such fluctuations. In COSI6, the fuel front-end path bears only one separated materials stock. Because of this, sophisticated reprocessing strategies are necessary in order to guarantee the physical properties (e.g. required isotopic composition) of the flow of separated material (e.g. plutonium).

A new approach of recipes was implemented in COSI7 in order to ease the reprocessing fine-tuning process. A COSI7 recipe consists in a model selecting and mixing separated materials from different buffer stocks, to provide a virtual buffer bearing desirable properties. However, this buffer does not feed directly the fabrication plant, but the equivalence model.

Let us consider the simplest example of MOX fuel fabrication from depleted uranium and two stocks of separated plutonium. Let $q_{1}$ and $q_{2}$ be two isotopic qualities in separated plutonium buffers, with $q_{i}$ defined as the ratio of the mass of fissile isotopes of Pu over the mass of $\mathrm{Pu}$ and ${ }^{241} \mathrm{Am}$ in stock $i$ :

$$
q_{i}=\left(m_{i}\left({ }^{239} P u\right)+m_{i}\left({ }^{241} P u\right)\right) /\left(m_{i}(P u)+m_{i}\left({ }^{241} A m\right)\right) .
$$

The COSI7 recipe also defines a target quality $q_{t}$ that must be reached in the fresh fuel. The behavior of the recipe is to pick the adequate mass of separated material mass in both stocks in order to reach the target quality $q_{t}$, thus replacing a series of micro-optimizations of the dataset. We remark that the total mass to pick is not pre-determined as it depends on the Pu content in fresh fuel which is evaluated by the equivalence model. Fig. 4 illustrates the recipe and equivalence process. First the recipe evaluates the fraction of separated materials $\alpha$ taken from the first stock as a function of $\left(q_{1}, q_{2}\right)$ such that the virtual recipe stock quality is $q_{t}$. Then the equivalence model evaluates the plutonium content $p$ as a function of the isotopic compositions of the virtual recipe stock and the depleted uranium stock.

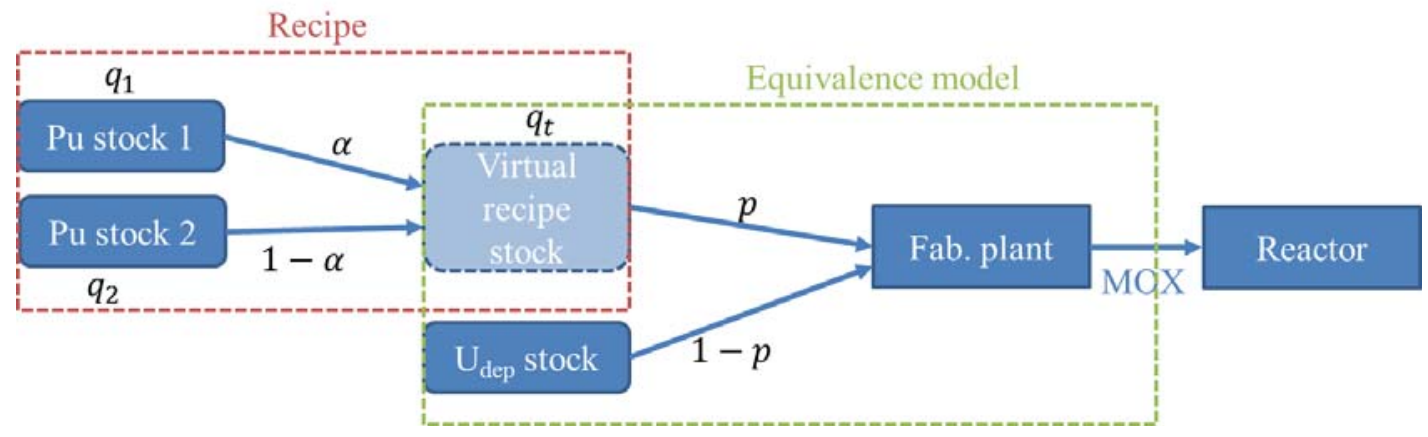

Figure 4. Recipe and equivalence processes in fresh fuel fabrication.

Fig. 5 illustrates the application of recipes to PWR MOX fuel fabrication using plutonium separated from both spent UOX fuel and spent MOX fuel. The plutonium quality in the Pu ex MOX feed is much lower than in the Pu ex UOX feed. Without using a recipe, the plutonium quality at MOX fabrication shows two distinct and generally unwanted trends:

- Short-term fluctuations due to the random nature of a batch-wise Pu feed;

- Long-term drift due to scenario dynamics (e.g. plutonium ageing).

Those trends are compensated using the recipe model. In this case, the recipe model setpoint is providing a $\mathrm{Pu}$ feed with $60 \%$ quality. 


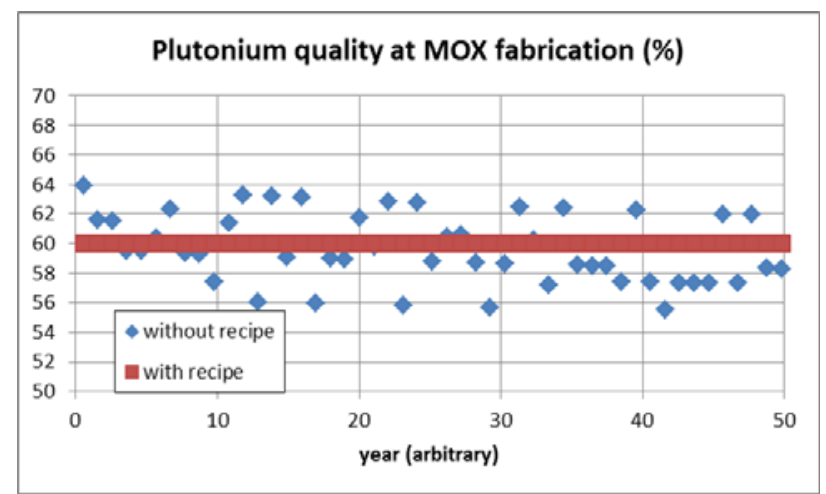

Figure 5. Application of recipe model to PWR MOX fuel fabrication.

Recipes are compatible with both on-demand reprocessing and fixed capacity reprocessing plant models. They can be adapted to match other criteria than the target quality such as isotopic composition, etc. They provide the user with a new tool to model constrained fabrication processes which cannot be modeled using previous versions of COSI6.

\section{CONCLUSIONS AND PERSPECTIVES}

The fuel cycle scenario code COSI7 was developed in order to answer demands from the fuel cycle community, falling into two groups: improving the user experience and providing new models to give more options to fine-tune scenarios.

Because the task of making and optimizing scenarios is very intensive in terms of engineering efforts, improving the user experience for scenario-makers was a priority. Many improvements over the previous version of COSI were implemented, including an internal dataset search engine, streamed post-processing functions, client-server architecture with shared cache. These improvements are expected to ease dataset implementation, scenario simulation and scenario analysis.

Several new physical models were also implemented in COSI7, designed in order to give the user the possibility to model even more complex yet realistic scenarios. For instance, the new implementation of recipes guarantees a given plutonium quality at fuel fabrication.

COSI7 supersedes COSI6 as the CEA reference nuclear fuel cycle simulation tool starting January 2020, and will be used to model the future nuclear fuel cycle scenarios in support of French stakeholders and decision-makers, including the scenarios associated with the French Multi-year Energy Plan (PPE).

\section{ACKNOWLEDGMENTS}

COSI7 development was funded by CEA project ACy/STECY.

\section{REFERENCES}

1. C. Coquelet-Pascal, M. Tiphine, G. Krivtchik, D. Freynet, C. Cany, R. Eschbach, C. Chabert, "COSI6: A Tool for Nuclear Transition Scenario Studies and Application to SFR Deployment Scenarios with Minor Actinide Transmutation", Nuclear Technology, Vol.192 (issue 2), pp.91-110 (2015)

2. G. Krivtchik, C. Coquelet-Pascal, P. Blaise, C. Garzenne, J. Le Mer, "Development of Surrogate Models of the Evolution Code for Uncertainty Propagation in Scenario Studies", Proc. Int. Conf. SNA \& MC 2013, Paris, France, October 27-31, 2013. 
3. G. Krivtchik, R. Eschbach, G. Martin, M. Tiphine, D. Freynet, R. Girieud, P. Miranda, "COSI6: A Tool for Nuclear Transition Scenario Studies", Technical Workshop on Nuclear Cycle Simulation (TW on NCS), Paris, France, July 6-8, 2016.

4. J.-M. Vidal et. al., "CESAR5.3 : A Code for Nuclear Fuel and Waste Characterization with Associated Qualification", Proc. Waste Management 2012 Conf. (WM2012), Phoenix, Arizona, February 26March 1, 2012. 